\title{
HPAanalyze: an R package that facilitates the retrieval and analysis of the Human Protein Atlas data
}

\author{
Anh Nhat Tran ${ }^{1 *}$, Alex M. Dussaq ${ }^{2}$, Timothy Kennell $\mathrm{Jr}^{3}$, Christopher D. Willey ${ }^{4}$ and Anita B. Hjelmeland ${ }^{1 *}$ (D)
}

\begin{abstract}
Background: The Human Protein Atlas (HPA) aims to map human proteins via multiple technologies including imaging, proteomics and transcriptomics. Access of the HPA data is mainly via web-based interface allowing views of individual proteins, which may not be optimal for data analysis of a gene set, or automatic retrieval of original images.

Results: HPAanalyze is an R package for retrieving and performing exploratory analysis of data from HPA. HPAanalyze provides functionality for importing data tables and xml files from HPA, exporting and visualizing data, as well as downloading all staining images of interest. The package is free, open source, and available via Bioconductor and GitHub. We provide examples of the use of HPAanalyze to investigate proteins altered in the deadly brain tumor glioblastoma. For example, we confirm Epidermal Growth Factor Receptor elevation and Phosphatase and Tensin Homolog loss and suggest the importance of the GTP Cyclohydrolase I/ Tetrahydrobiopterin pathway. Additionally, we provide an interactive website for non-programmers to explore and visualize data without the use of $\mathrm{R}$.
\end{abstract}

Conclusions: HPAanalyze integrates into the R workflow with the tidyverse framework, and it can be used in combination with Bioconductor packages for easy analysis of HPA data.

Keywords: Human protein atlas, Proteomics, Visualization, Software

\section{Background}

The Human Protein Atlas (HPA) is a comprehensive resource for exploration of the human proteome which contains a vast amount of proteomics and transcriptomics data generated from antibody-based tissue micro-array profiling and RNA deep-sequencing [1-7]. The program has generated protein expression profiles in human non-malignant tissues, cancers, and cell lines with cell type-specific expression patterns via an innovative immunohistochemistrybased approach. These profiles are accompanied by a large collection of high-quality histological staining images that are annotated with clinical data and quantification. The database also includes classification of proteins into both functional classes (such as transcription factors or kinases)

\footnotetext{
* Correspondence: trannhatanh89@gmail.com; hjelmea@uab.edu

'Department of Cell, Developmental and Integrative Biology, University of Alabama at Birmingham, THT 948, 1900 University Blvd, Birmingham, AL 35294, USA

Full list of author information is available at the end of the article
}

and project-related classes (such as candidate genes for cancer). Starting from version 4.0, the HPA includes subcellular localization profiles based on confocal images of immunofluorescent, stained cells. Together, these data provide a detailed picture of protein expression in human cells and tissues, facilitating tissue-based diagnostic and research.

Data from the HPA are freely available via proteinatlas.org, allowing scientists to access and incorporate the data into their research. Previously, the R package hpar has been created for fast and easy programmatic access of HPA data [8]. Here, we introduce HPAanalyze, an R package that aims to simplify exploratory data analysis from those data, as well as provide other functions complementary to hpar.

\section{Implementation}

HPAanalyze is an $\mathrm{R}$ software package with GPL-3 license that is designed for easy retrieval and exploratory 
analysis of data from HPA. HPAanalyze allows users to quickly import data tables and xml files from HPA and provides a visual summary of the data. All staining images available in HPA can also be downloaded. Data can be obtained for single proteins or a protein set for pathway analysis. jsHPAanalyze is a JavaScript software suite with a GPL-3 license designed to create an interface in which non-programmers can simulate the R software package environment.

\section{The different HPA data formats}

The HPA project provides data via two main mechanisms: Full datasets in the form of downloadable compressed Tab-Separated Value (TSV) files are available as well as individual entries in Extensible Markup Language (XML), Resource Description Framework (RDF), and TSV formats. The full downloadable datasets include normal tissue, pathology (cancer), subcellular location, RNA gene, and RNA isoform data. For individual entries, the XML format is the most comprehensive: it provides information on the target protein, antibodies, and a summary of each tissue. Also provided are detailed data from each sample including clinical information, immunohistochemistry (IHC) scoring, and image download links.

\section{HPAanalyze overview}

HPAanalyze is designed to fulfill three main tasks: (1) import, subsetting and export downloadable datasets; (2) visualization of downloadable datasets for exploratory analysis; and (3) facilitation of work with individual XML files (Fig. 1). This package aims to serve researchers with little programming experience, while also allowing power users to utilize the imported data as desired.

\section{Obtaining HPAanalyze}

The stable version of HPAanalyze is available via Bioconductor and can be installed with the following code:

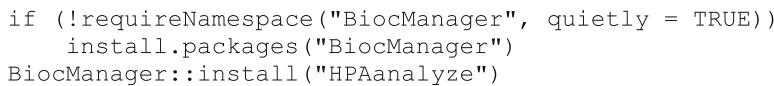

The development version of HPAanalyze is available on Github can be installed with the following code:

if (!requirenamespace ("devtools", quietly = TRUE)) install.packages ("devtools")

devtools: :install github ("trannhatanh89/HPAanalyze")

\section{Full dataset import, subsetting and export}

The hpaDownload function downloads full datasets from HPA and imports them into $\mathrm{R}$ as a list of data frames (the "tibble"/ tbl_df variant commonly used in the tidyverse framework [9]). Data frames can subsequently be subset with hpaSubset and exported into XLSX, CSV or TSV formats with hpaExport. The standard object allows the imported data to be further processed in a traditional $\mathrm{R}$ workflow. The ability to quickly subset and export data gives researchers the option to use other non-R downstream tools, such as GraphPad for creating publication-quality graphics, or share a subset of data containing only proteins of interest.

\section{Visualization}

With the intent to aid exploratory analysis, the hpaVis function family takes the output of hpaDownload (or hpaSubset) and provides quick visualization of the data. Nevertheless, the standard ggplot [10] object output of these functions gives users the option to further customize the plots for publication. All hpaVis functions share the same syntax for arguments: subsetting, specifying colors, and opting to use custom themes.

The first release of the HPAanalyze package includes three functions: hpaVisTissue for normal tissue samples, hpaVisPatho for the pathology/cancer samples, and hpaVisSubcell for subcellular localization data. All operations of this function family can be easily accessed through the umbrella function hpaVis.

\section{Individual XML import and image downloading}

The hpaXml function family imports and extracts data from individual XML entries from HPA. The hpaXmlGet function downloads and imports data as an " $x m l_{-}$ document"/ "xml_node" object, which can subsequently be processed by other hpaXml functions. The XML format from HPA contains a wealth of information that may not be covered by this package. However, users can extract any data of interest from the imported XML file using the $x m l 2$ package.

In the first release, HPAanalyze includes four functions for data extraction from HPA XML files: hpaXmlProtClass for protein class information, hpaTissueExprSum for summary of protein expression in tissue, hpaXmlAntibody for a list of antibodies used to stain for the protein of interest, and hpaTissueExpr for complete and detailed data from each sample including clinical data and IHC scoring. hpaTissueExprSum and hpaTissueExpr provide download links to obtain relevant staining images, with the former function also providing the option to automate the downloading process. Similar to the hpaVis family, all functionalities of this family may also be accessed through the simple umbrella function hpaXml.

\section{Compatibility with hpar Bioconductor package}

HPAanalyze was designed to be compatible and complementary to other existing software packages. Table 1 shows the different capibilities of HPAanalyze and hpar, 


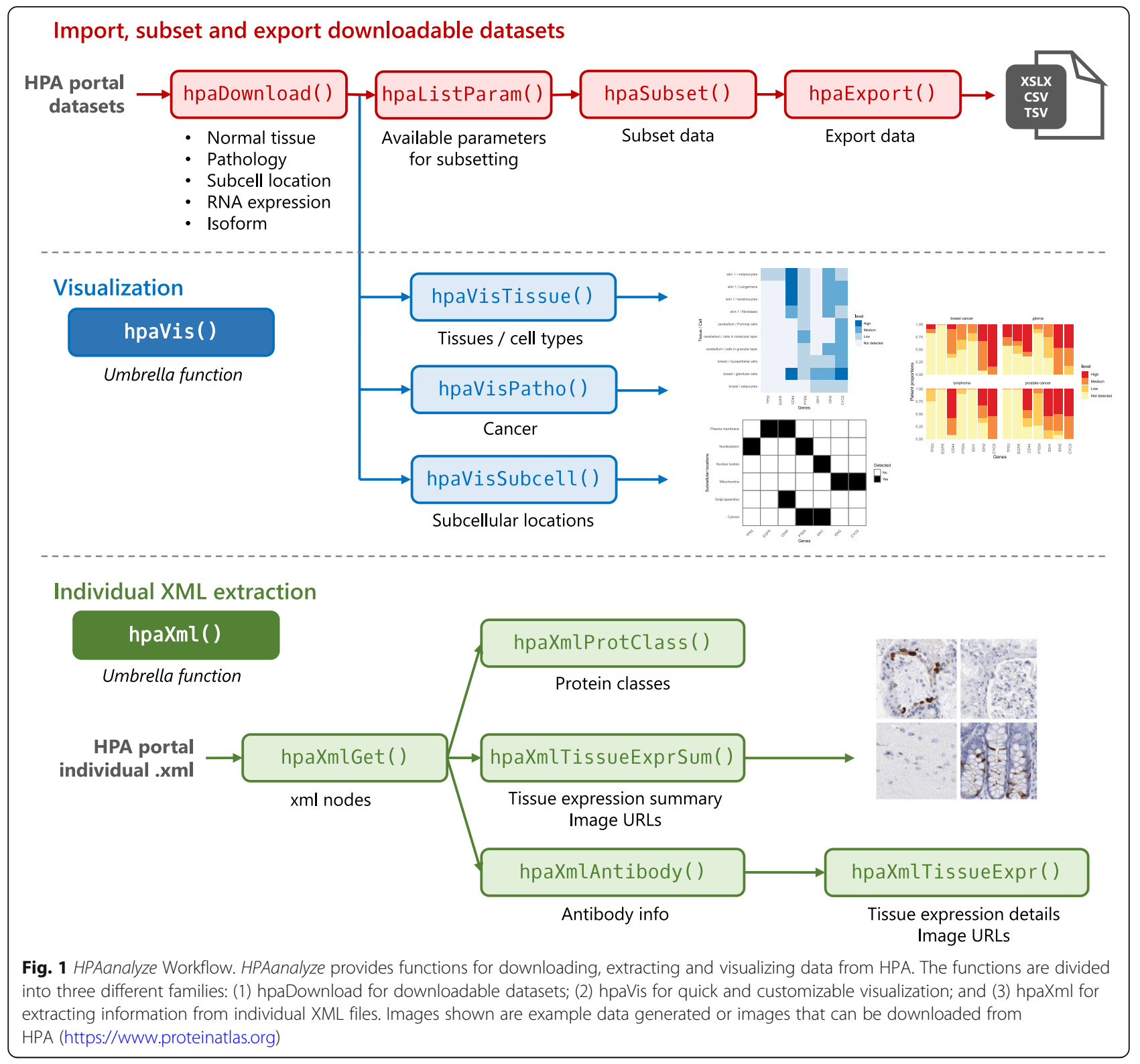

Table 1 Complementary functionality between hpar and HPAanalyze

\begin{tabular}{lll}
\hline Functionality & hpar & HPAanalyze \\
\hline Datasets & Included in package & Download from server or import from hpar \\
Query & Ensembl id & HGNC symbol and Ensembl id \\
Data version & One stable version & Latest by default, option to download older \\
Release info & Access via functions & Not applicable \\
View relevant browser page & Via getHPA function & Not applicable \\
Visualization & Not applicable & Exploratory via hpaVis functions \\
XML & Not applicable & Download and import via hpaXml functions \\
Histology image & View by loading browser page & Extract links via hpaXml functions \\
\hline
\end{tabular}


a Bioconductor package optimized for fast acquisition of subsets of HPA data.

\section{Typical workflows and sample codes}

The HPAanalyze package can be loaded with the following code:

$$
\text { library (HPAanalyze) }
$$

\section{Working with HPA downloadable datasets}

Using HPAanalyze, a typical workflow with HPA downloadable datasets consists of the following steps:

1. Download and import data into R with hpaDownload.

2. View available parameters for subsetting with hpaListParam.

3. Subset data with hpaSubset.

4. Optional: Export data with hpaExport (Fig. 1).

The following code can be used to download the histology datasets (normal tissue, pathology, and subcellular location).

$$
\text { data <- hpaDownload (downloadList = 'histology') }
$$

The output of the code shows that data can be subset by normal tissue types, normal cell types, cancer types, and subcellular location. The "normal_tissue" dataset contains information about protein expression profiles in human tissues based on IHC staining. The "pathology" dataset contains information about protein expression profiles in human tumor tissue based on IHC staining with the number of patients annotated for four staining levels together with log-rank $p$ values for survival/mRNA correlation. The "subcellular_location" dataset contains information about subcellular localization of proteins based on immunofluorescence (IF) staining of normal cells.

hpaListParam function prints a list of available parameters that can be used to subset the downloaded datasets. Below are the first three items in each group:

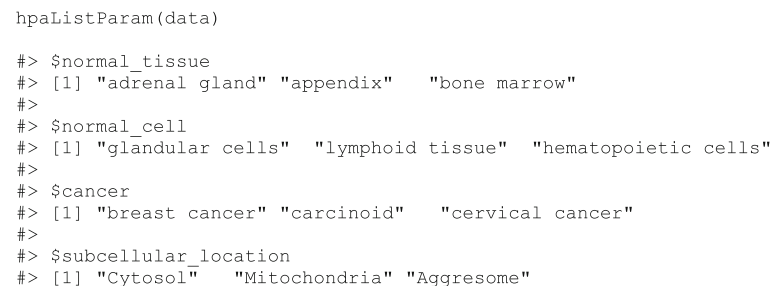

Based on the information, the downloaded data may be subset based on genes, tissues, cells and subcellular locations of interest. As an example, the following code filters the datasets for MKI67 (Ki67), breast tissue, and breast cancer.

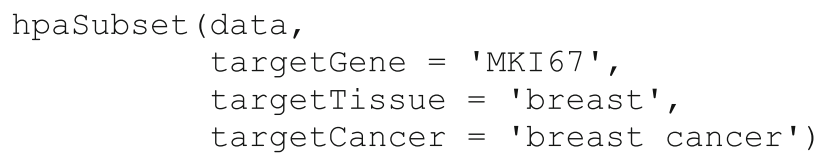

The results (below) showed that Ki67 is expressed at non-detectable-to-medium levels in normal breast tissue, but medium-to-high levels in breast cancer. The data also indicated, with high reliability, that Ki67 is expressed at high levels in the nuclear bodies, nucleoli and nucleus.

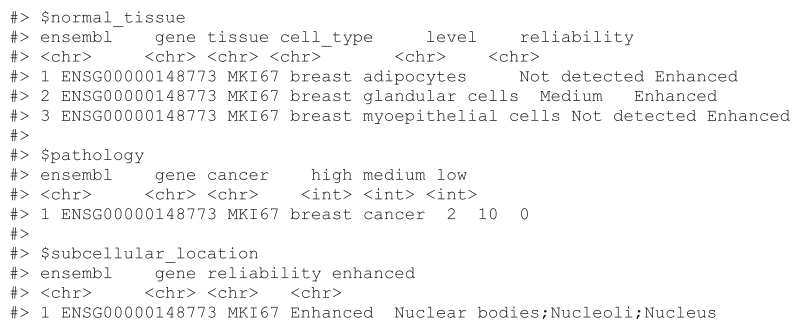

We next sought to facilitate the downstream analysis of data using a non-R software as well as the storage of data subsets for reproducible research. To accomplish this goal, the HPAanalyze package included the $h p a E x$ port function. The hpaExport function exports data into Excel file format, with each sheet for a dataset. As an example, the code to export the above Ki67 data and generate an .xlsx file called 'ki67.xlsx' is as noted below.

hpaExport (data $=$ ki67_data, fileName $=" k i 67 "$, fileType $=$ "xlsx")

\section{Visualization with the hpaVis function family}

With the goal of aiding exploratory analysis of a group of target proteins, HPAanalyze provides the ability to quickly visualize data from downloaded HPA datasets with the hpaVis function family (Fig. 1). These functions maybe particularly useful for gaining insights into pathways or gene signatures of interest.

The hpaVis functions share a common syntax, where the input is the object generated by hpaDownload or hpaSubset. Depending on the function, the target arguments allows the user to choose to visualize vectors of genes, tissue, cell types, etc. All hpaVis functions generate standard ggplot 2 plots, which allow further customization of colors and themes. Currently, the normal tissue, pathology, and subcellular localization data can be visualized.

hpaVisTissue generates a "heatmap", in which the expression of proteins of interest as measured with quantified IHC staining is plotted for each cell type of each tissue (Fig. 2a). hpaVisPatho generates an array of column graphs showing the expression of proteins of interest in each cancer (Fig. 2b). hpaVisSubcell generates 


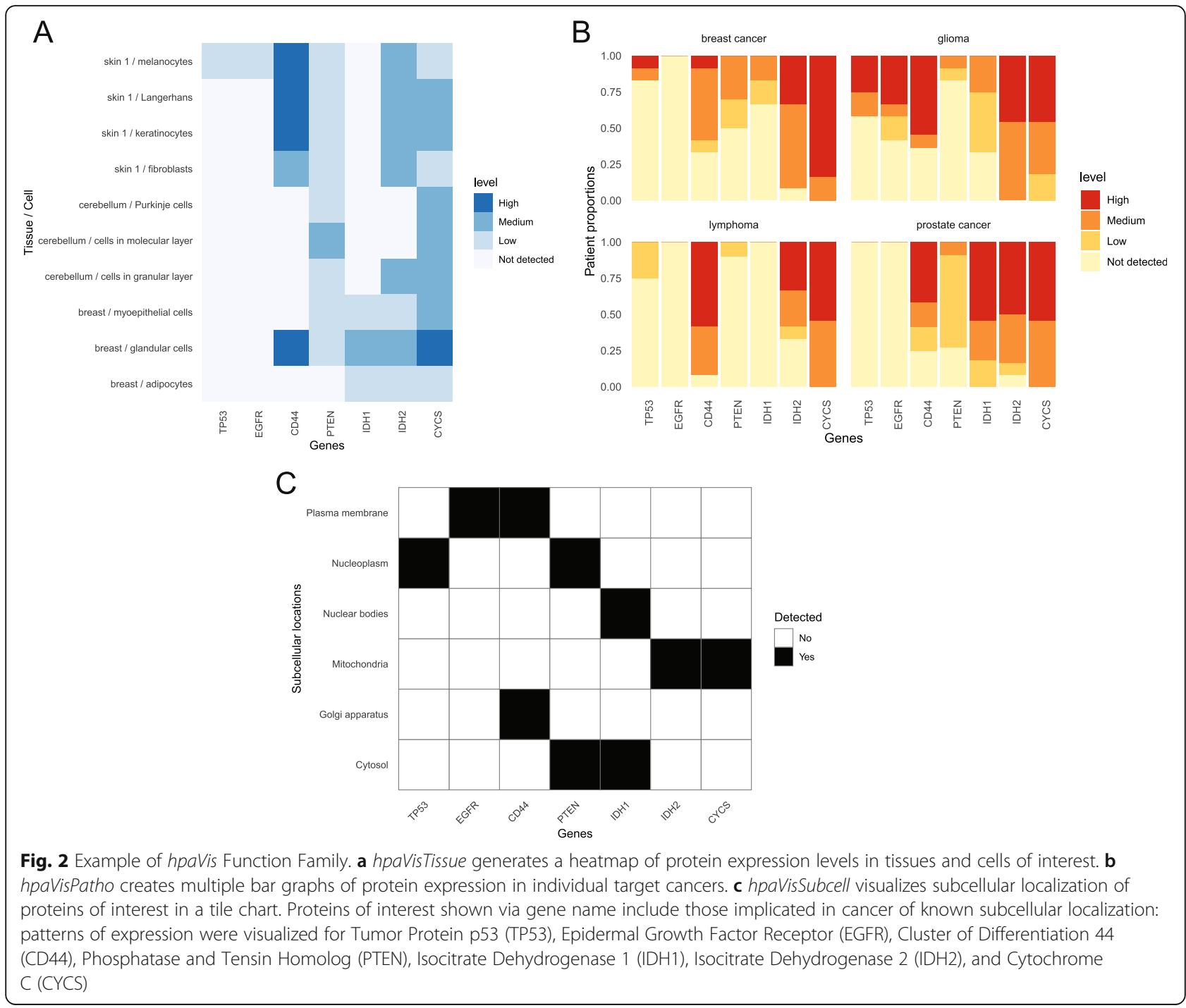

a tile chart showing the subcellular localization of proteins of interest (Fig. 2c).

Working with individual XML files for each target protein The hpaXml function family supports importing and extracting data from individual XML files provided by HPA for each protein. A typical workflow for use of XML files includes the following steps:

1. Download and import XML file with hpaXmlGet.

2. Extract the desired information with other hpaXml functions.

3. Download images of histological stains as currently supported by the hpaXmlTissurExpr and hpaXmlTissueExprSum functions (Fig. 1).

The hpaXmlGet function takes one HGNC symbol or Ensembl id (starting with ENSG) and imports the perspective XML file into $R$. This function calls the $x m l 2::$ read_xml function under the hood, hence the resulting object may be processed further with functions from the $x m l 2$ package if desired. The protein class of a queried protein can be extracted from the imported XML with hpaXmlProtClass. The function hpaXmlTissueExprSum extracts the summary of expression of a protein of interest in normal tissue. The output of this function is (1) a string containing a one-sentence summary, and (2) a data frame of all tissues in which the protein was positively stained and images of those tissues.

The XML files are the only format of HPA programmatically accessible data that contains information about each antibody and each tissue sample used in the project. hpaXmlAntibody extracts the antibody information and returns a data frame with one row for each antibody. hpaXmlTissueExpr extracts information about all samples for each antibody above and returns a list of 
data frames. If an antibody has not been used for IHC staining, the returned data frame will be empty. Each data frame contains clinical data (patientid, age, sex), tissue information (snomedCode, tissueDescription), staining results (staining, intensity, location) and one imageUrl for each sample.

\section{jsHPAnalyze: availability and use}

We implemented the hpaVis function family in JavaScript, and this is available on GitHub at: https://github. com/adussaq/jsHPAanalyze. A tutorial on how to utilize the tool is available on YouTube at: https://youtu.be/ 9mZj7NJKiAE. We utilized bootstrap 4.3.1 for the page layout, apex charts 3.6.12 for figure creation, and Dexie 2.0.4 for data caching. To utilize the tool a user needs to limit the list of genes to less than 50 . This can be done by selecting sections from each of the charts and by manually selecting genes by name. Once the user has limited the list, all three figures can be produced from the hpaVis function family.

\section{Results}

To demonstrate the potential uses of HPAanalyze, we performed example case studies. Each case study was chosen based on the availability of a body of literature that could be used to validate functionality by demonstrating how the resulting data might confirm or complement cancer research.

\section{Case study 1: Glioma pathway alteration at the protein level}

As further detailed below, previous studies elucidating pathway alterations in glioblastoma (GBM) have identified frequent amplifications, deletions and mutations of certain genes belonging to Phosphatidylinositol-3-kinase (PI3K)/Mitogen-activated Protein Kinase (MAPK), p53 and Retinoblastoma Protein $(\mathrm{Rb})$ pathways. The level of these proteins in normal brain (hippocampus and cerebral cortex) (Fig. 3a) and glioma (Fig. 3b) was therefore visualized with HPAanalyze. These data were acquired by quantification of IHC stained specimens, so levels of mutant proteins may not be represented. Furthermore, the cancer dataset contains data for all glioma: information about each specific cancer grade is only available via further examination of individual antibodies, which is outside of the scope of this case study.

As a positive control, we first evaluated the expression of Glial Fibrillary Acidic Protein (GFAP), a marker for astrocytes/glial cells. In the normal dataset, GFAP expression was found only on glial cells, suggesting that a false positive is unlikely (Fig. 3a). In glioma datasets, GFAP was found to be expressed at mostly medium to high levels (Fig. 3b), which is also consistent with the literature.
According to the TCGA data for GBM patients, approximately $90 \%$ of patients have alterations in the PI3K/MAPK pathway: Epidermal Growth Factor Receptor (EGFR), Platelet-derived Growth Factor Receptor Alpha (PDGFRA), and PI3K genes are frequently amplified or mutated to gain function (approximately 57, 10 and 25\%, respectively), while Phosphatase and Tensin Homolog (PTEN) is deleted or mutated in $41 \%$ of patients [11]. Data from HPAanalyze supports this pattern, although the proportions are not identical (Fig. 3a-b). Differences can be attributed to the distinctions between target molecules (DNA/mRNA in TCGA versus protein in HPA) and the number of specimens. One example of the difference can be observed with data regarding $\mathrm{v}$-Raf Murine Sarcoma Viral Oncogene Homolog B (BRAF). BRAF is only amplified/mutated in $2 \%$ of TCGA patients, but it is expressed at medium to high levels in all glioma specimens in HPA (Fig. 3b).

The p53 pathway is altered in $86 \%$ of GBM patients, with amplification of MDM2 (7.6\%) and MDM4 (7.2\%) leading to the inhibition of $\mathrm{p} 53$, which is also highly mutated [11]. The amplification of MDM2 and MDM4 is reflected at protein levels in HPA: MDM2 is expressed at high levels in all patients and MDM4 at medium levels in most patients (Fig. 3b). Similarly, the Rb pathway inhibitor CDK4 was found to be amplified in $14 \%$ of patient samples and confirmed by the stark contrast between normal and cancerous samples in HPA (Fig. 3a-b). The protein CDK4 is not detected in any normal brain cell, while it is present at some level in most glioma samples. These data confirm that HPAanalyze may be useful for comparison of normal and tumor tissue in order to identify or validate molecules of interest with altered expression in cancer.

\section{Case study 2: PTEN's novel function through chromatin- associated complexes}

PTEN is known as a key tumor suppressor which is frequently mutated in GBM [11]. Canonically, the protein functions as a phosphatase to dephosphorylate phosphatidylinositol $(3,4,5)$-trisphosphate $\left(\mathrm{PIP}_{3}\right)$, which leads to inhibition of Akt signaling [12]. Akt is central to many hallmarks of cancer by promoting cell survival via inhibition of the apoptotic protein Bad, overcoming cell cycle arrest, facilitating glucose metabolism, inhibiting autophagy via regulation of the lysosomal biogenesis controller TFEB, and promoting tumor angiogenesis [13].

Since $\mathrm{PIP}_{3}$ is a phospholipid that resides on the plasma membrane [13], PTEN was once thought to act solely in the cytoplasm. However, a recently published study demonstrated that PTEN also forms complexes with the histone chaperone DAXX and the histone variant H3.3, modulating chromatin association to regulate oncogene expression. This effect is independent of PTEN enzymatic 


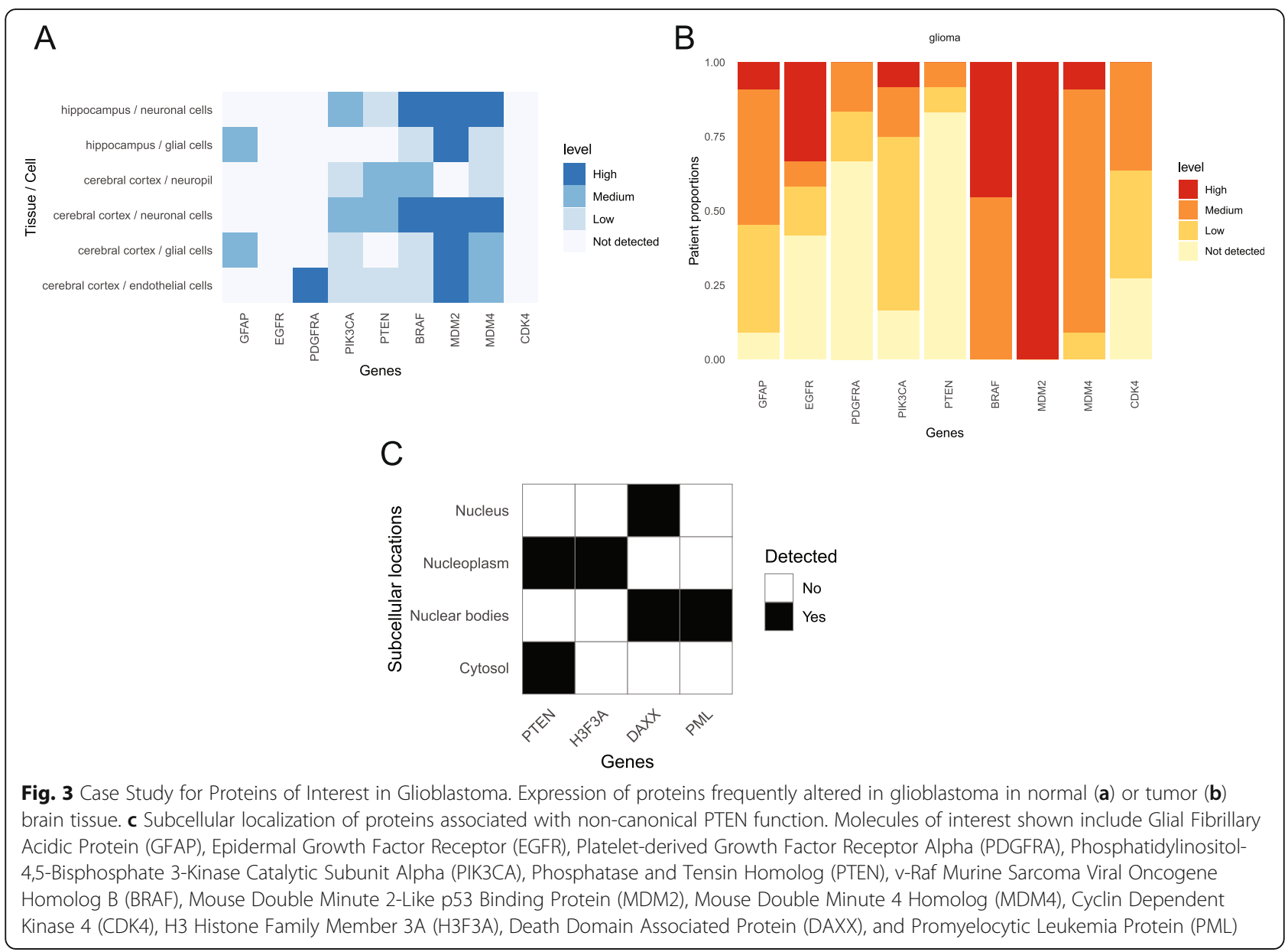

activity [14]. Congruent with these data, we noted that PTEN was present in both the cytosol and the nucleus (Fig. 3c) in HPA data, suggesting a non-canonical function for PTEN. The subcellular localization of DAXX and H3.3, as well as PML (which interacts with DAXX and regulates PTEN), further corroborate the newly discovered model of PTEN-DAXX-H3.3 gene regulation (Fig. 3c).

HPA subcellular localization information for individual proteins is acquired via immunofluorescent staining of human cell lines [3]. Therefore, the data do not account for various physiological conditions that may relocate proteins nor do the data directly provide evidence of protein-protein interactions. A query of HPA should always be followed by a confirmation study to ensure the validity of the results in any cell type or cancer of interest. Nevertheless, HPAanalyze offers a powerful approach to quickly explore curated and validated antibody-based protein expression data.

Case study 3: Protein expression of GTP Cyclohydrolase I (GCH1)/tetrahydrobiopterin (BH4) pathway members

Summarized datasets regarding the expression of one protein may not be sufficient to understand the potential role of a pathway in normal or cancer tissue. We recently defined a role for GTP Cyclohydrolase I (GCH1), the first and rate limiting enzyme in the tetrahydrobiopterin (BH4) pathway, as an important regulator of glioblastoma growth [15]. The GCH1/BH4 pathway can regulate the production of reactive species, which can be pro- or anti-tumorigenic depending on a number of factors which we and others have reviewed [16]. In addition to $\mathrm{GCH} 1$ and the final product $\mathrm{BH} 4$, the de novo pathway also involves 6-pyruvoyltetrahydropterin synthase (PTS) and sepiapterin reductase (SPR), the latter of which has been known to be targeted by multiple well-established sulfa drugs [17]. BH4 can also be produced via the salvage pathway in which the oxidized product $\mathrm{BH} 2$ is converted back to BH4 by dihydrofolate reductase (DHFR) [18].

Using HPAanalyze, we confirmed our published finding that GCH1 was elevated in glioma in comparison to nontumor brain tissue (Fig. 4a-b, Additional files 1 and 2). HPAanalyze indicated that $\mathrm{GCH} 1$ was not expressed in normal brain (cerebellum, cortex, hippocampus or caudate) (Fig. 4a). However, GCH1 was detected in glioma samples, and increased with tumor grades (Fig. 4b). Our analysis using the HPA datasets showed that, while SPR 


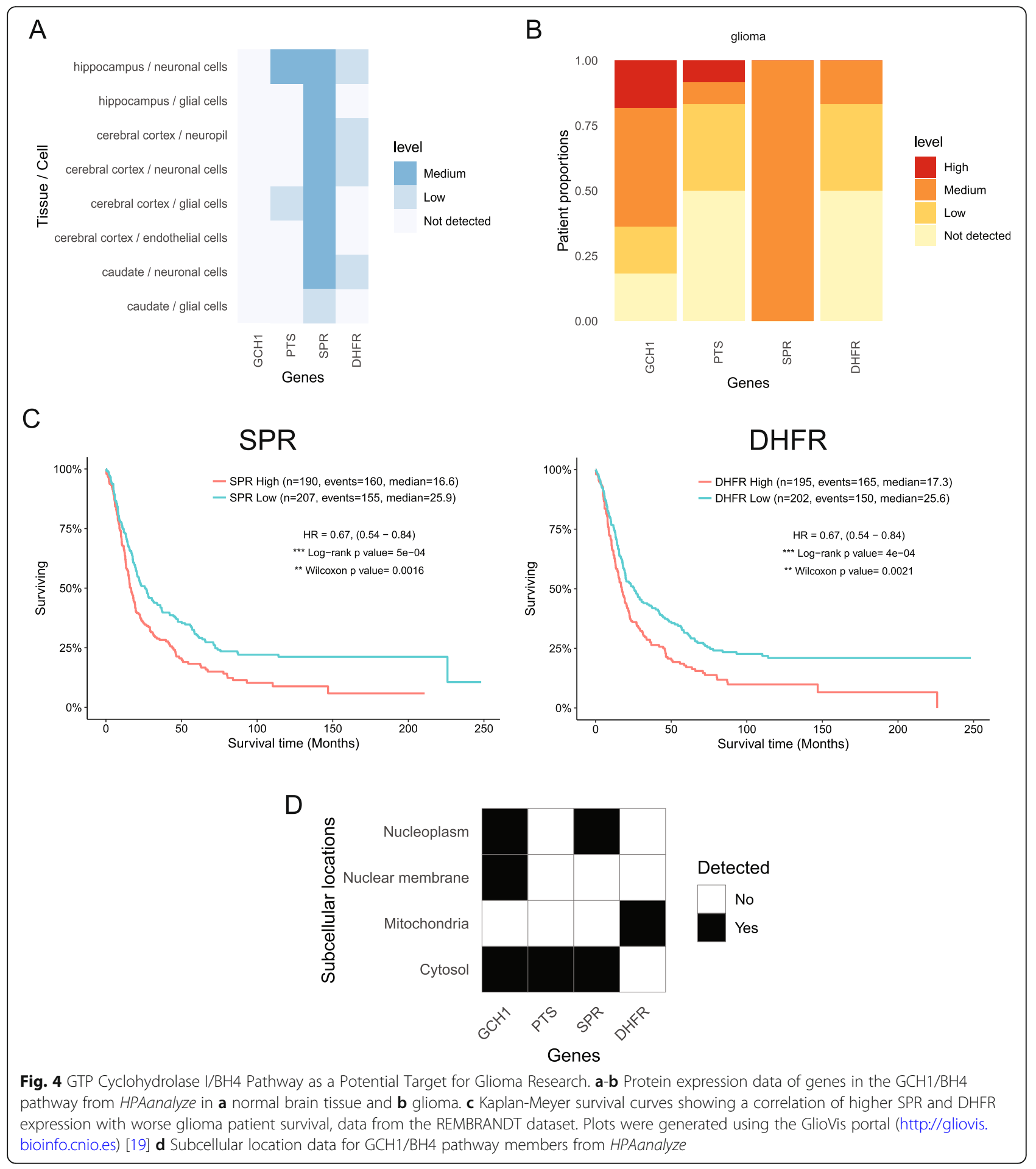

expression was relatively consistent between normal brain and tumor tissues, the other three members of the pathway were expressed at higher levels in glioma tumors than in normal cells (Fig. 4a-b). Survival analysis also demonstrated that, similar to our published findings with GCH1, elevated expression of SPR or DHFR correlated with worse survival of glioma patients (Fig. 4c,
Additional files 3 and 4). Together, these data strengthen the notion that the BH4 pathway is important in glioma growth and suggest additional targets for therapeutic intervention, including those with readily available small molecule inhibitors.

The hpaVisSubcell function of HPAanalayze revealed an interesting aspect of the $\mathrm{GCH} 1 / \mathrm{BH} 4$ pathway protein 
expression that is worthy of additional investigation. All members of the de novo biosynthesis pathway were expressed in the cytosol where they are expected to function as enzymes to produce $\mathrm{BH} 4$. However, GCH1 and SPR were also present in the nucleus (Fig. 4d), which may suggest additional roles as in transcriptional regulation.

\section{Case study 4: Glucose transporter 3 (GLUT3/ SLC2A3) in normal brain and glioma}

To explore the capability of HPAanalyze to retrieve details of proteins of interest from HPA, we focused on GLUT3 (encoded by the gene SLC2A3) which facilitates the transport of glucose through cell plasma membranes. Together with other proteins in its family, GLUT3 plays an important role in regulating the metabolism in mammalian cells. In many cancers, including glioma, metabolic abnormality has been shown to promote tumor growth and maintenance [20]. In fact, GLUT3 inhibitors have been investigated as potential therapy for glioma [21]. Using the hpaSubset function, we found that GLUT3 expression was not detected in glial cells in the brain, while about a third of the glioma patients in the HPA datasets had GLUT3 expression in their tumors (Additional files 1 and 2).

To acquire more details, we used the hpaXmlGet function to download the corresponding XML file for GLUT3. The hpaXmlAntibody function revealed that the
HPA program had used two different antibodies to stain for GLUT3. Using the hpaXmlTissueExpr function, we were able to extract the full record of every staining available for both antibodies, including clinical data, staining quantifications, and links to the original images (Additional files 1 and 2). From there, we were able to filter the records and download GLUT3 staining images for normal brain tissue and glioma samples (Fig. 5). Those high-quality images may be further assessed by pathologists for information not available from HPA.

\section{Conclusions}

We report the development of the $\mathrm{R}$ package HPAanalyze, which we believe will be highly useful for investigators interested in visualizing the expression data from HPA for signaling pathways. Using our $R$ package HPAanalyze, we are able to retrieve, visualize and export data from the HPA program. Additionally, we created jsHPAanalyze which allows for non-programmers, with nothing more than a modern browser, to be able to create the visualizations described in this publication. We have new functionality compared to other available packages in that we can visualize the data as well as quickly download histological images of interest. Although it is a programmatic approach, which requires basic R programming skills, HPAanalyze was built with ease of use and reproducibility in mind, which makes the workflow and syntax very simple and straight-
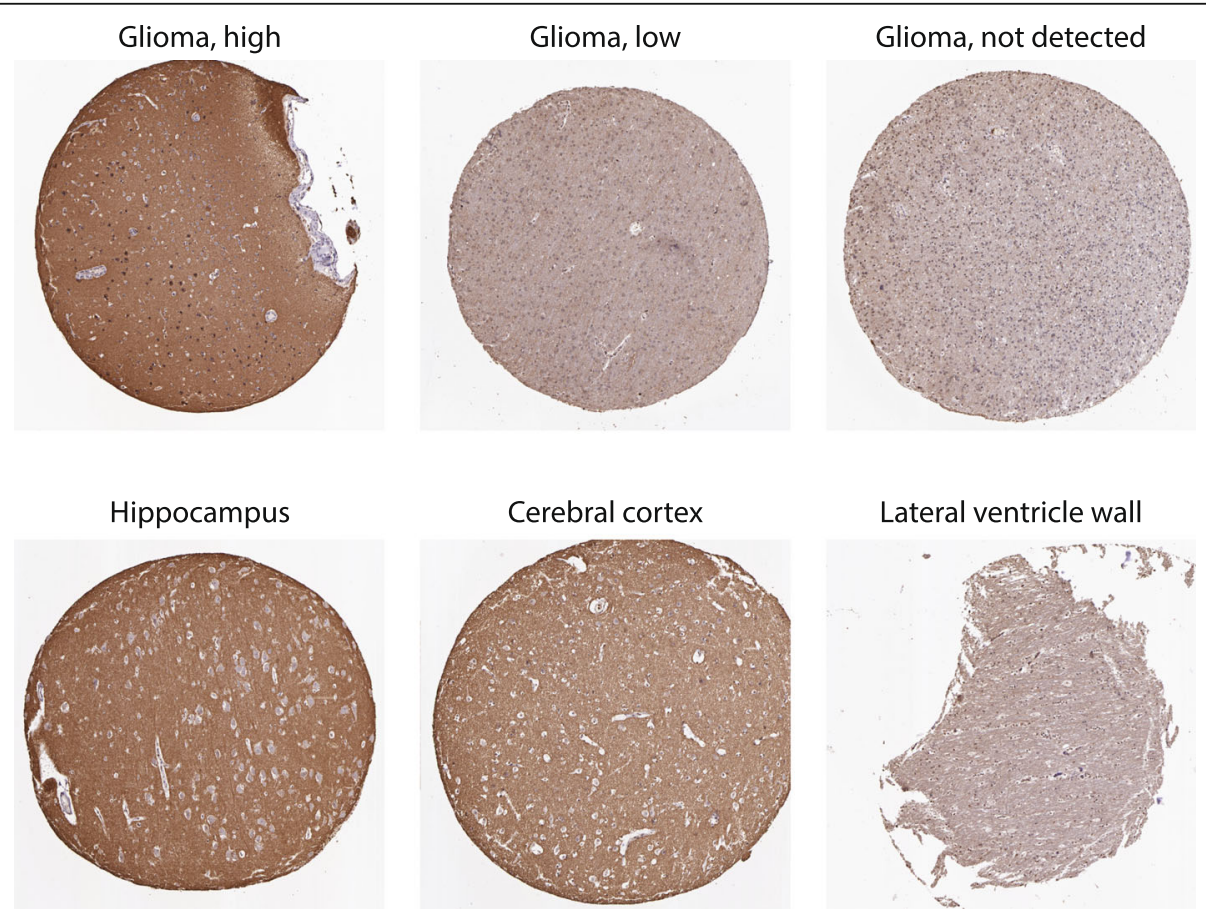

Fig. 5 Expression of GLUT3 in the brain. Representative images of GLUT3 staining in normal brain and glioma tissues. The images are downloaded as detailed in the Additional files 1 and 2 using HPAanalyze and additional R codes 
forward. With the case studies, we have also demonstrated how HPAanalyze can be easily integrated into different areas of research to identify new targets or provide more evidence for a working hypothesis. This software package is highly supportive of our research, and we plan to update it with new features and ensure future compatibility with the HPA program.

\section{Availability and requirements HPAanalyze}

- Project name: HPAanalyze

- Project home page: https://github.com/ trannhatanh89/HPAanalyze

- Operating system(s): All platforms where R is available, including Windows, Linux, OS X

- Programming language: $\mathrm{R}$

- Other requirements: R 3.5.0 or higher, and the R packages dplyr, openxlsx, ggplot2, readr, tibble, $\mathrm{xml} 2$, tidyr, stats, utils, hpar, gridExtra

- License: GPL-3

- Any restrictions to use by non-academics: Freely available to everyone

\section{jsHPAanalyze}

- Project name: jsHPAanalyze

- Project home pages: https://github.com/adussaq/ jsHPAanalyze

- Operating system(s): All platforms where a modern browser is available, including Windows, Linux, OS X

- Programming language: JavaScript

- Other requirements: Modern browser such as Chrome or Firefox

- License: GPL-3

- Any restrictions to use by non-academics: Freely available to everyone

\section{Additional files}

Additional file 1: $\mathrm{R}$ Codes for Figs. 2, 3, 4 and $5 \mathrm{html}$. Supplemental material for Figs. 2, 3, 4 and $5 \mathrm{html}$ format. Input parameters for HPAanalyze to generate Figs. 2, 3, 4 and 5 are shown. (HTML $626 \mathrm{~kb}$ )

Additional file 2: $\mathrm{R}$ Codes for Figs. 2, 3, 4 and $5 \mathrm{rmd}$. Supplemental material for Figs. 2, 3, 4 and 5 rmd format. Input parameters for HPAanalyze to generate Figs. 2, 3, 4 and 5 are provided. (RMD $8 \mathrm{~kb}$ )

Additional file 3: SPR. Supplemental material for Fig. 4. Primary data in GlioVis used to generate the survival curves demonstrating that elevated SPR mRNA expression correlates with poor glioma patient survival. (CSV 26 kb)

Additional file 4: DHFR. Supplemental material for Fig. 4. Primary data in GlioVis used to generate the survival curves demonstrating that elevated DHFR mRNA expression correlates with poor glioma patient survival. (CSV 26 kb)

\section{Abbreviations}

BRAF: v-Raf Murine Sarcoma Viral Oncogene Homolog B; CD44: Cluster of Differentiation 44; CDK4: Cyclin Dependent Kinase 4; CSV: Comma-Separated
Values; CYCS: Cytochrome C; DAXX: Death Domain Associated Protein; DHFR: Dihydrofolate Reductase; EGFR: Epidermal Growth Factor Receptor: GBM: Glioblastoma; GCH1: GTP Cyclohydrolase I; GFAP: Glial Fibrillary Acidic Protein; GLUT3/SLC2A3: Glucose Transporter 3; Solute Carrier Family 2 Member 3; H3F3A: H3 Histone Family Member 3A; HGNC: HUGO Gene Nomenclature Committee; HPA: Human Protein Atlas; IDH1: Isocitrate Dehydrogenase 1; IDH2: Isocitrate Dehydrogenase 2; IF: Immunofluorescence; IHC: Immunohistochemistry; MAPK: Mitogen-activated Protein Kinase; MDM2: Mouse Double Minute 2-Like p53 Binding; MDM4: Mouse Double Minute 4 Homolog; MTX: Methotrexate; PDGFRA: Platelet-derived Growth Factor Receptor Alpha; PI3K: Phosphatidylinositol-3-kinase;

PIK3CA: Phosphatidylinositol-4,5-Bisphosphate 3-Kinase Catalytic Subunit Alpha; PML: Promyelocytic Leukemia Protein; PTEN: Phosphatase and Tensin Homolog; PTS: 6-pyruvoyltetrahydropterin synthase; Rb: Retinoblastoma Protein; RDF: Resource Description Framework; SPR: Sepiapterin Reductase; TCGA: The Cancer Genome Atlas; TP53: Tumor Protein p53; TSV: TabSeparated Value; XML: Extensible Markup Language

\section{Acknowledgements}

Not applicable.

\section{Authors' contributions}

ANT created the R package, analyzed data, generated figures, and wrote the manuscript. AMD, TKJr, and CDW assisted with package validation and manuscript revision. AMD generated the website tool. ABH supervised the project, assisted with data analysis, and wrote the manuscript. All authors read and approved the final manuscript.

\section{Funding}

We appreciate the support of the National institutes of Health R01 NS104339 and R21 NS096531 funds from the Department of Cell, Developmental and Integrative Biology at the University of Alabama at Birmingham. Funders did not have any role in the development of the R package or the design of the case studies, nor did the funders influence the conclusions.

\section{Availability of data and materials}

All data analyzed during this study are publicly available at https://www. proteinatlas.org. The R package is available at https://github.com/ trannhatanh89/HPAanalyze.

Ethics approval and consent to participate

Not applicable.

\section{Consent for publication}

Not applicable.

\section{Competing interests}

The authors declare that they have no competing interests.

\section{Author details}

'Department of Cell, Developmental and Integrative Biology, University of Alabama at Birmingham, THT 948, 1900 University Blvd, Birmingham, AL 35294, USA. ${ }^{2}$ Department of Pathology, University of Alabama at Birmingham, 121 Shelby Biomedical Research Building, Birmingham, AL 35294, USA. ${ }^{3}$ Department of Genetics, University of Alabama at Birmingham, 121 Shelby Biomedical Research Building, Birmingham, AL 35294, USA. ${ }^{4}$ Department of Radiation Oncology, University of Alabama at Birmingham, 176 Facility Building, Birmingham, AL 35294, USA.

Received: 6 February 2019 Accepted: 27 August 2019

Published online: 09 September 2019

\section{References}

1. Berglund L, Björling E, Oksvold P, Fagerberg L, Asplund A, Szigyarto CA-K, Persson A, Ottosson J, Wernérus H, Nilsson P, et al. A genecentric Human Protein Atlas for expression profiles based on antibodies. Mol Cell Proteomics. 2008;7(10):2019-27.

2. Pontén $\mathrm{F}$, Jirström K, Uhlen M. The Human Protein Atlas--a tool for pathology. J Pathol. 2008;216(4):387-93. 
3. Thul PJ, Åkesson L, Wiking M, Mahdessian D, Geladaki A, Ait Blal H, Alm T, Asplund A, Björk L, Breckels LM, et al. A subcellular map of the human proteome. Science. 2017;356(6340).

4. Uhlén $M$, Björling E, Agaton C, Szigyarto CA-K, Amini B, Andersen E, Andersson A-C, Angelidou P, Asplund A, Asplund C, et al. A human protein atlas for normal and cancer tissues based on antibody proteomics. Mol Cell Proteomics. 2005;4(12):1920-32.

5. Uhlén M, Fagerberg L, Hallström BM, Lindskog C, Oksvold P, Mardinoglu A, Sivertsson Å, Kampf C, Sjöstedt E, Asplund A, et al. Proteomics. Tissue-based map of the human proteome. Science. 2015;347(6220):1260419.

6. Uhlen $M$, Oksvold P, Fagerberg L, Lundberg E, Jonasson $K$, Forsberg $M$, Zwahlen M, Kampf C, Wester K, Hober S, et al. Towards a knowledge-based Human Protein Atlas. Nat Biotechnol. 2010;28(12):1248-50.

7. Uhlen M, Zhang C, Lee S, Sjöstedt E, Fagerberg L, Bidkhori G, Benfeitas R, Arif M, Liu Z, Edfors F, et al. A pathology atlas of the human cancer transcriptome. Science. 2017;357(6352).

8. Gatto L. hpar: Human Protein Atlas in R. In., 1.22.2 edn: Bioconductor; 2018.

9. Wickham H, Grolemund G. R for data science : import, tidy, transform, visualize, and model data. 1st ed. Sebastopol: O'Reilly; 2016.

10. Wickham H. Ggplot2 : elegant graphics for data analysis. New York: Springer; 2009.

11. Brennan Cameron W, Verhaak Roel GW, McKenna A, Campos B, Noushmehr H, Salama Sofie R, Zheng S, Chakravarty D, Sanborn JZ, Berman Samuel H, et al. The Somatic Genomic Landscape of Glioblastoma. Cell. 2013;155(2): 462-77.

12. Koul D. PTEN signaling pathways in glioblastoma. Cancer Biol Ther. 2008; 7(9):1321-5.

13. Auger KR, Serunian LA, Soltoff SP, Libby P, Cantley LC. PDGF-dependent tyrosine phosphorylation stimulates production of novel polyphosphoinositides in intact cells. Cell. 1989;57(1):167-75.

14. Benitez JA, Ma J, D'Antonio M, Boyer A, Camargo MF, Zanca C, Kelly S, Khodadadi-Jamayran A, Jameson NM, Andersen M, et al. PTEN regulates glioblastoma oncogenesis through chromatin-associated complexes of DAXX and histone H3.3. Nat Commun. 2017:8:15223.

15. Tran AN, Walker K, Harrison DG, Chen W, Mobley J, Hocevar L, Hackney JR, Sedaka RS, Pollock JS, Goldberg MS, et al. Reactive species balance via GTP cyclohydrolase I regulates glioblastoma growth and tumor initiating cell maintenance. Neuro Oncol. 2018;20(8):1055-67.

16. Libby CJ, Tran AN, Scott SE, Griguer C, Hjelmeland AB. NOS Expression and NO Function in Glioma and Implications for Patient Therapies. Antioxid Redox Signal. 2017;26(17):986-99.

17. Haruki H, Pedersen MG, Gorska Kl, Pojer F, Johnsson K. Tetrahydrobiopterin Biosynthesis as an Off-Target of Sulfa Drugs. Science. 2013;340(6135):987-91

18. Crabtree MJ, Tatham AL, Hale AB, Alp NJ, Channon KM. Critical role for tetrahydrobiopterin recycling by dihydrofolate reductase in regulation of endothelial nitric-oxide synthase coupling: relative importance of the de novo biopterin synthesis versus salvage pathways. J Biol Chem. 2009; 284(41):28128-36

19. Bowman RL, Wang Q, Carro A, Verhaak RG, Squatrito M. GlioVis data portal for visualization and analysis of brain tumor expression datasets. Neuro Oncol. 2017;19(1):139-41.

20. Libby CJ, Tran AN, Scott SE, Griguer C, Hjelmeland AB. The pro-tumorigenic effects of metabolic alterations in glioblastoma including brain tumor initiating cells. Biochim Biophys Acta Rev Cancer. 2018;1869(2):175-88.

21. Libby CJ, Zhang SX, Benavides GA, Scott SE, Li YJ, Redmann M, Tran AN, Otamias A, Darley-Usmar V, Napierala M, et al. Identification of Compounds That Decrease Glioblastoma Growth and Glucose Uptake in Vitro. ACS Chem Biol. 2018;13(8):2048-57.

\section{Publisher's Note}

Springer Nature remains neutral with regard to jurisdictional claims in published maps and institutional affiliations.

Ready to submit your research? Choose BMC and benefit from:

- fast, convenient online submission

- thorough peer review by experienced researchers in your field

- rapid publication on acceptance

- support for research data, including large and complex data types

- gold Open Access which fosters wider collaboration and increased citations

- maximum visibility for your research: over $100 \mathrm{M}$ website views per year

At BMC, research is always in progress.

Learn more biomedcentral.com/submissions 